\title{
Evaluation of a tuberculous abscess on the right side of the diaphragm with contrast-enhanced computed tomography: A case report
}

\author{
PENG DONG $^{1}$, JING-JING CHEN ${ }^{2}$ and XI-ZHEN WANG ${ }^{1}$ \\ ${ }^{1}$ Medical Imaging Center, The Affiliated Hospital of Weifang Medical University, Weifang, Shandong 261031; \\ ${ }^{2}$ Department of Radiology, The Affiliated Hospital of Qingdao University School of Medicine, \\ Qingdao, Shandong 266000, P.R. China
}

Received November 3, 2015; Accepted February 22, 2016

DOI: $10.3892 / \mathrm{mco} .2016 .863$

\begin{abstract}
We herein investigate the case of a patient with a tuberculous diaphragmatic abscess confirmed by pathology. The patient underwent plain computed tomography (CT) examination of the chest and contrast-enhanced abdominal CT examination. The abscess appeared as hypodense mass with thick and irregular wall, which was enhanced on the contrast-enhanced CT images. The shape of the mass resembled an irregular double convex lens. No enlarged lymph nodes were detected on the CT images. The presence of a tuberculous diaphragmatic abscess should be suspected in patients with a diaphragmatic hypodense mass with enhanced thick walls, even when there is absence of enlarged lymph nodes on the CT images.
\end{abstract}

\section{Introduction}

Mycobacterium tuberculosis (TB) infection remains a challenge to human health $(1,2)$. Although abdominal TB is uncommon, the peritoneum is commonly involved in patients with abdominal TB infection $(3,4)$. Charoensak et al $(4)$ reported that certain cases with tuberculous peritonitis may mimic other diseases, which represents a diagnostic challenge for clinicians.

A few cases with intraperitoneal tuberculous abscesses have been previously reported, whereas diaphragmatic tuberculous abscess (DTA) is particularly rare (4-6). The aim of this case report was to gain some experience regarding the computed tomography (CT) findings in this DTA case, which

Correspondence to: Professor Peng Dong, Medical Imaging Center, The Affiliated Hospital of Weifang Medical University, Shenglidong Street, Weifang, Shandong 261031, P.R. China

E-mail: dongpeng98021@sina.com

Key words: tuberculosis, diaphragmatic abscess, X-ray, computed tomography was pathologically confirmed, with the hope that it will be contributory to the comprehensive CT diagnosis of DTAs.

\section{Case report}

The present study was reviewed and approved by the Institutional Review Board of The Affiliated Hospital of Weifang Medical University. The patient provided written informed concent was provided to the publication of this case.

A 22-year-old male patient presented with night sweats, easy fatigability and obscure right upper abdominal pain for 60 days. The clinical examination of the patient did not identify an abdominal palpable mass, and there was no history of lung TB.

Plain CT scanning of the chest was performed using the GE Bright Speed 16-detector spiral CT scanner (GE Medical Systems, Chicago, IL, USA) with the following parameters: $120 \mathrm{KV}, 130 \mathrm{mAs}$ and $5 \mathrm{~mm}$ slice width. Contrast-enhanced abdominal CT scanning was performed using the Siemens Somatom Sensation Cardiac 64-slice spiral CT scanner (Siemens, Munich, Germany) with the following parameters: $120 \mathrm{KV}, 200-390 \mathrm{mAs}$ and $5 \mathrm{~mm}$ slice width. The patient received intravenous Ultravist (300 mgI/ml; Bayer Schering Pharma AG, Berlin, Germany) administered as a bolus, at an injection rate of $3.0 \mathrm{ml} / \mathrm{sec}$. Oral contrast material (water) was administered.

The CT images revealed a hypodense diaphragmatic mass, sized $6.0 \times 4.5 \times 3.5 \mathrm{~cm}$, pushing on the parenchyma of the liver. The shape of the mass resembled an irregular double convex lens based on the right side of the diaphragm. The wall of the mass exhibited irregular thickening with enhancement. Gas collection or calcification were not detected within the mass (Figs. 1-3). The right side of the diaphragm was discontinuous. The right retroperitoneal space and the adjacent pleura were invaded, with marginally increased thickness of the subpleural fatty tissue (Fig. 4). No enlarged lymph nodes were detected in the mediastinum, root of the mesentery or upper para-aortic region. No tuberculous lesion was detected on the bilateral lung fields (Fig. 5).

Surgery was performed. The mass involved the right side of the diaphragm and adhered to the liver. The small and large 


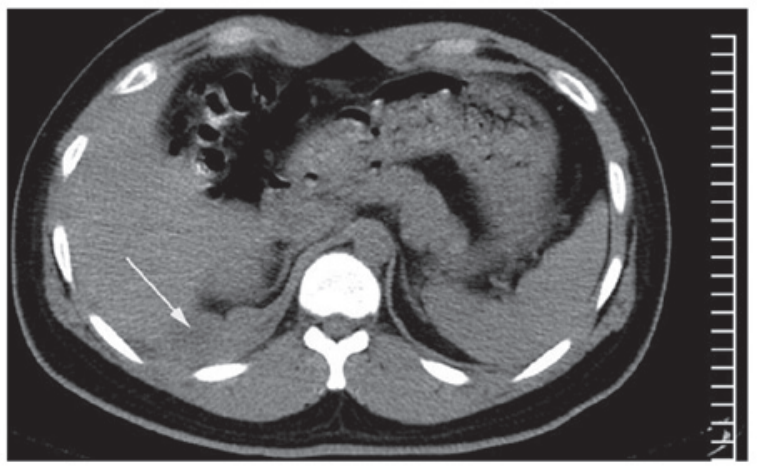

Figure 1. Axial plain computed tomography image showing a hypodense diaphragmatic mass (white arrow) pushing on the hepatic parenchyma. The shape of the mass resembled an irregular double convex lens.
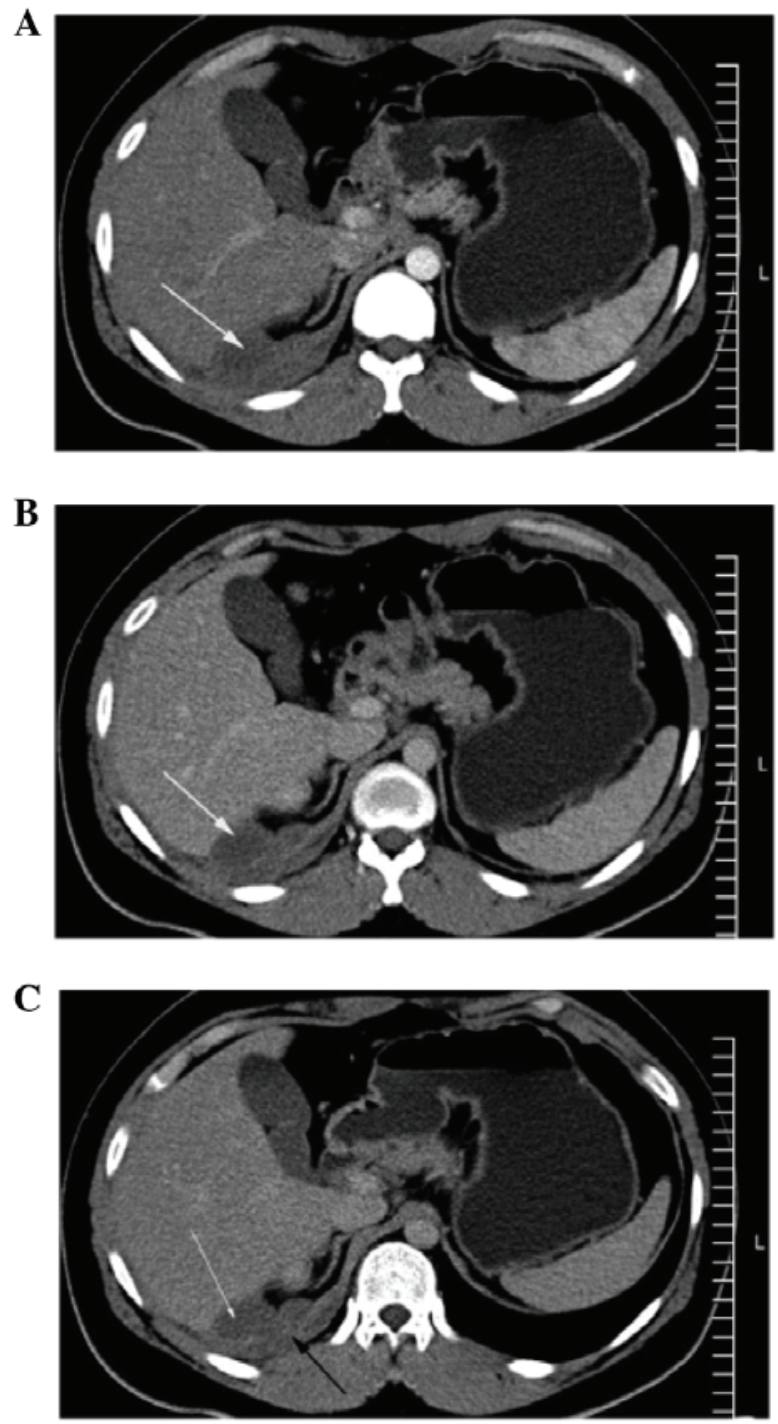

Figure 2. Axial computed tomography images during the (A) arterial, (B) venous and (C) delayed phases, showing the wall of the mass as being irregularly thick with enhancement (white arrows). The diaphragm was discontinuous (black arrow).

bowel were normal. The pathological diagnosis was that of caseous TB. After being treated with anti-tuberculosis drugs, the patient fully recovered and was discharged from hospital.
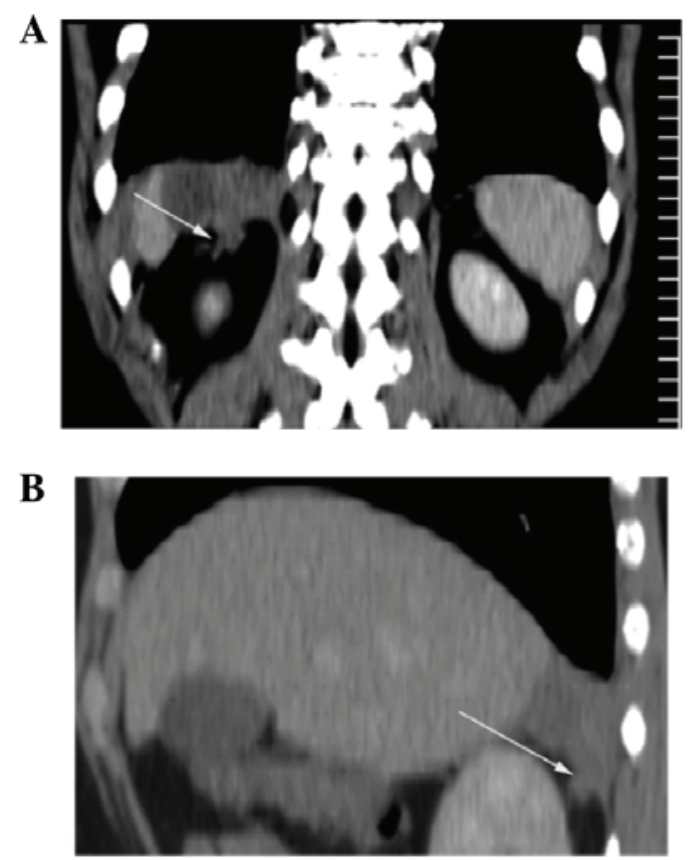

Figure 3. (A) Coronal and (B) sagittal contrast-enhanced computed tomography images showing invasion of the right retroperitoneal space (white arrows).

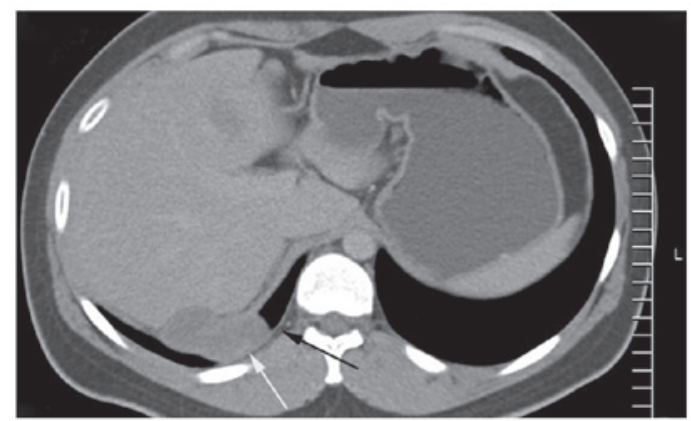

Figure 4. Axial computed tomography image showing invasion of the adjacent pleura (white arrow). The subpleural fatty tissue was thicker compared with that of the contralateral side (black arrow). No enlarged lymph nodes were identified.

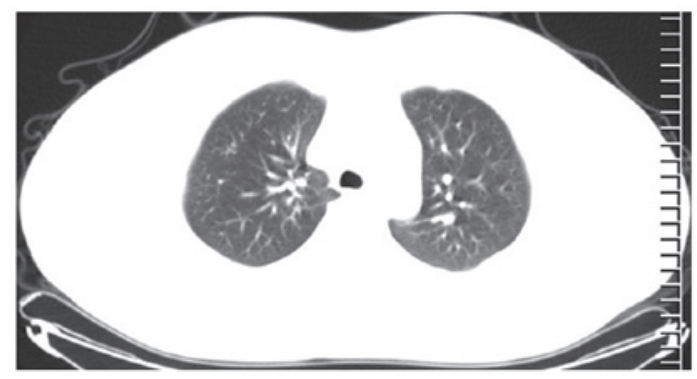

Figure 5. Axial computed tomography image did not reveal tuberculous lesions of the bilateral lung field.

\section{Discussion}

Tuberculous lesions are associated with a variable spectrum of medical imaging manifestations, and they may mimic 
other diseases (6-9). It is important to familiarize with the CT manifestations of tuberculous lesions to ensure accurate CT diagnosis.

Abdominal TB commonly spreads or disseminates by the following routes: i) Intestinal TB spreading to the lymph nodes; ii) hematogenous dissemination; and iii) direct extension of adjacent tuberculous lesions (8). Only a small proportion of patients with abdominal TB also suffer from pulmonary TB (7). It has been reported that multifocal TB may occur secondary to lympho-hematogenous dissemination after the initial infection (10). In our patient, the adjacent pleura and retroperitoneal space were invaded, whereas no enlarged lymph nodes or other tuberculous lesions were detected. This indicates that the spreading route of this case of DTA may be hematogenous dissemination.

The reasons for the rarity of skeletal muscle TB may be as follows: i) High levels of lactic acid; ii) not enough reticuloendothelial tissue; iii) not enough lymphatic tissue; and iv) rich blood supply $(11,12)$. Thus, the abovementioned reasons may help to explain the rarity of the DTA.

In the present study, the wall of the DTA was irregularly thickened with permanent enhancement, which was similar to the cases reported in the literature (6). The right retroperitoneal space displayed fibrous strands neighboring the mass, which also conformed to inflammatory changes. In this case, the shape of the abscess (irregular double convex lens), the simultaneous involvement of the pleural cavity and retroperitoneal space with a discontinuous diaphragm on CT images may help with the localization and diagnosis.

Differential diagnosis. Most inflammations of the diaphragm result from direct extension from adjacent lesions (13), whereas lung cancer may directly invade the diaphragm (14). In this case, no lesions were detected within the lung field.

Malignant pleural mesothelioma may involve the diaphragm, and trans-diaphragmatic extension may be detected on CT examination (15). A rind-like pleura, with a thickness of $>10 \mathrm{~mm}$ is crucial for the diagnosis of malignant pleural mesothelioma (16). Increased thickness of the subpleural fatty tissue was the sign for the diagnosis of benign pleural lesion on CT images (16). In the present case, the subpleural fatty tissue adjacent to the diaphragmatic mass was thicker compared with that of the contralateral side, which conformed to the diagnosis of a benign pleural lesion.

It may be difficult to distinguish diaphragmatic from abdominal tumors, particularly in the case of tumors arising from the right side of the diaphragm (17). In the present case, no abnormal signs were detected within the liver parenchyma and peritoneum.

Primary tumors of the diaphragm are rare, and the most common benign lesions are diaphragmatic cysts (18). The CT density of the diaphragmatic mesothelial cyst is similar to that of water, and the margin is well-defined without enhancement (19), which was different from the findings in this case.

In summary, DTA, despite its rarity, should be suspected in patients with a diaphragmatic hypodense mass exhibiting enhanced thick wall, even in the absence of enlarged lymph nodes on CT images.

\section{References}

1. Lee WK, Van Tonder F, Tartaglia CJ, Dagia C, Cazzato RL, Duddalwar VA and Chang SD: CT appearances of abdominal tuberculosis. Clin Radiol 67: 596-604, 2012.

2. LoBue PA, Enarson DA and Thoen TC: Tuberculosis in humans and its epidemiology, diagnosis and treatment in the United States. Int J Tuberc Lung Dis 14: 1226-1232, 2010.

3. Khan R, Abid S, Jafri W, Abbas Z, Hameed K and Ahmad Z: Diagnostic dilemma of abdominal tuberculosis in non-HIV patients: An ongoing challenge for physicians. World J Gastroenterol 12: 6371-6375, 2006.

4. Charoensak A, Nantavithya P and Apisarnthanarak P: Abdominal CT findings to distinguish between tuberculous peritonitis and peritoneal carcinomatosis. J Med Assoc Thai 95: 1449-1456, 2012.

5. Dong P, Chen JJ, Wang XZ and Wang YQ: Intraperitoneal tuberculous abscess: Computed tomography features. World J Radiol 7: 286-293, 2015.

6. Dong P, Wang B and Sun YQ: Tuberculous abscess in the hepatoduodenal ligament: Evaluation with contrast-enhanced computed tomography. World J Gastroenterol 14: 2284-2287, 2008.

7. Akhan $\mathrm{O}$ and Pringot J: Imaging of abdominal tuberculosis. Eur Radiol 12: 312-323, 2002.

8. Yang ZG, Min PQ, Sone S, He ZY, Liao ZY, Zhou XP, Yang GQ and Silverman PM: Tuberculosis versus lymphomas in the abdominal lymph nodes: Evaluation with contrast-enhanced CT. AJR Am J Roentgenol 172: 619-623, 1999.

9. Yang GY, Zhao D, Zhang WZ, Meng J, Li J, Li XH and Wan HF: Role of ultrasound evaluation for the diagnosis and monitoring of thyroid tuberculosis: A case report and review of the literature. Oncol Lett 9: 227-230, 2015.

10. Al-Tawfiq JA: Multifocal systemic tuberculosis: The many faces of an old nemesis. Med Sci Monit 13: CS56-CS60, 2007.

11. Chewoolkar V, Bichile L and Patel H: Pyomyositis with multifocal osteomyelitis - an uncommon presentation of skeletal tuberculosis. J Assoc Physicians India 57: 706, 2009.

12. Neogi DS, Bandekar SM and Chawla L: Skeletal muscle tuberculosis simultaneously involving multiple sites. J Pediatr Orthop B 22: 167-169, 2013.

13. Ye Y, Yang Z, Li H, Deng W, Li Y and Guo Y: MDCT features and anatomic-pathology in right thoracic-abdominal junctional region diseases. Sheng Wu Yi Xue Gong Cheng Xue Za Zhi 28: 255-259, 2011 (In Chinese).

14. Yokoi K, Tsuchiya R, Mori T, Nagai K, Furukawa T, Fujimura S, Nakagawa K and Ichinose Y: Results of surgical treatment of lung cancer involving the diaphragm. J Thorac Cardiovasc Surg 120: 799-805, 2000.

15. Nickell LT Jr, Lichtenberger JP III, Khorashadi L, Abbott GF and Carter BW: Multimodality imaging for characterization, classification and staging of malignant pleural mesothelioma. Radiographics 34: 1692-1706, 2014.

16. Metintas M, Ucgun I, Elbek O, Erginel S, Metintas S, Kolsuz M, Harmanci E, Alatas F, Hillerdal G, Ozkan R and Kaya T: Computed tomography features in malignant pleural mesothelioma and other commonly seen pleural diseases. Eur J Radiol 41: 1-9, 2002.

17. Asahi Y, Kamiyama T, Nakanishi K, Yokoo H, Tahara M, Usui A, Funakoshi T, Sato M, Sasaki A, Matsuno Y, et al: Chondroma of the diaphragm mimicking a giant liver tumor with calcification: Report of a case. Surg Today 44: 2361-2365, 2014.

18. Kim MP and Hofstetter WL: Tumors of the diaphragm. Thorac Surg Clin 19: 521-529, 2009.

19. Esparza Estaún J, González Alfageme A and Sáenz Bañuelos J: Radiological appearance of diaphragmatic mesothelial cysts. Pediatr Radiol 33: 855-858, 2003. 